\title{
Central Anticholinergic Syndrome Following Excessive Mydriatic Use in an Eight-Year-Old Patient
}

\author{
(D) Nazan Kaymaz¹, (D) Mehmet Erdem Uzun², (D) Fatih Battal1, (D) Yusuf Güzel1, (D) Hakan Aylanç¹ \\ ${ }^{1}$ Çanakkale Onsekiz Mart University Hospital, Department of Pediatrics, Çanakkale, Turkey \\ 2University of Health Sciences Turkey, Bursa Yüksek ihtisas Training and Resarch Hospital, Clinic of Child Psychhiatry, Bursa, Turkey
}

Cite this article as: Kaymaz N, Uzun ME, Battal F, Güzel Y, Aylanç H. Central Anticholinergic Syndrome Following Excessive Mydriatic Use in an Eight-Year-Old Patient. Trends in Pediatrics 2021;2(4):170-172

\begin{abstract}
Cyclopentolate hydrochloride, belonging to a class of drugs known as anticholinergics, is an ophthalmic solution frequently used in ophthalmology clinics because of its cycloplegic and mydriatic effects in both refractions as well as fundus examinations. After application, the maximum cycloplegic effect begins approximately after 30-60 min, which can further continue up to $24 \mathrm{~h}$. Due to a dose-dependent relationship, cyclopentolate hydrochloride's side effects can be observed both in an ocular and systemic manner which may occur if solutions more than $0.5 \%$ concentration are used. Herein, we report central anticholinergic syndrome due to the administration of excessive amounts of cyclopentolate hydrochloride in a child.
\end{abstract}

Keywords: Child, mydriatics, cyclopentolate, ophthalmic solutions, dilatation

\section{INTRODUCTION}

A refractory ophthalmological examination is extremely crucial for children aiming at an early diagnosis of likely refractive errors and potential eye disorders that might hinder a child's normal growth. Cyclopentolate hydrochloride eye drops are commonly used as potential cycloplegic and mydriatic agents to assist in several refractive evaluations and are systemically absorbed from the conjunctiva as well as the nasal mucosa. Although rare, several side effects may develop as a result of inadequate dose individualization and excessive systemic absorption leading to the active ingredient's unwanted bioavailability. Since intoxication symptoms appear 30 min after the drug's administration, they usually persist for 4-8 hours and then resolve without significant sequelae, ${ }^{1}$ but certain patients may experience few symptoms like ataxia, dysarthria, disorientation, hallucination, euphoria, unreasonable laughter, agitation, increased motor activity, confusion, and delirium during this period. ${ }^{2}$ Herein, we describe an eight-year-old male child with central anticholinergic syndrome caused by an excessive dosage of cyclopentolate hydrochloride.

\section{CASE REPORT}

An eight-year-old male patient, without any previous systemic health issues, was referred to our pediatric emergency clinic with symptoms of disorganized speech, hallucinations, and periods of hyperactivity. After obtaining the patient's medical history, it was revealed that the patient who previously wore glasses due to hyperopia, underwent an annual follow-up examination in an ophthalmology outpatient clinic that had prescribed cyclopentolate hydrochloride to create mydriasis half an hour before the examination. Additionally, his mother was also explained the entire drug dispensing procedure and thereby was asked to administer the eye drops to the patient, but due to a gross misunderstanding of the drug dosage, it was applied incorrectly as a repetition of one drop in each eye with

N. Kaymaz: 0000-0002-3962-4799; ME. Uzun: 0000-0003-4311-2670; Y. Güzel: 0000-0002-1241-3561; H. Aylanç: 0000-0002-8907-3809 
an interval of five minutes for approximately one hour until the examination time thus, a total of 12 drops was instilled in each eye. The patient visited our pediatric emergency clinic for a follow-up visit due to aggravated adverse symptoms including disorganized speech, hallucinations, and hyperactive periods, caused by copious drug administration. His vital signs were as follows: body temperature, $37.3{ }^{\circ} \mathrm{C}$; blood pressure, 110/60 mmHg; heart rate, 95 beats/minute; respiratory rate, 20 breaths/minute; and oxygen saturation, $97 \%$ at room air. $\mathrm{He}$ had dry oral mucosa and a rash on his face with dilated pupils and a negative response to light reflex. Additionally, the patient also exhibited various symptoms like dysarthria, ataxic gait, disorientation of place, time and person, significantly increased motor activity as well as psychomotor agitation. It was observed that he was talking too fast in meaningless context accompanied by visual hallucinations and a lack of attention while other systemic and neurological physical examination findings were not detected. There was no history suggesting infection or fever, central nervous system infection, and no history suggesting electrolyte disturbances that may might occur due to fluid loss such as vomiting or/and diarrhea. The patient was monitored and followed up in the emergency department after considering the diagnosis of central anticholinergic syndrome due to excessive usage of cyclopentolate hydrochloride. Normal sinus rhythm was observed in the electrocardiogram while the blood pressure and heart rate evaluations were normal. All possible complications of mydriatic intoxication were properly monitored. As his symptoms returned to normal after 4 hours, he was discharged after 8 hours of follow-up.

\section{DISCUSSION}

In this case report, an eight-year-old boy diagnosed as having central anticholinergic syndrome following excessive cyclopentolate hydrochloride dosage as a mydriatic agent for a regular ophthalmic examination was presented. Abnormal examination findings found in the patient were dry oral mucosa, prolonged mydriatic state, lack of attention, increased motor activity, and visual hallucinations.

Systemic side effects of cyclopentolate hydrochloride can be classified into two groups as neuropsychiatric and nonneuropsychiatric. Non-neuropsychiatric side effects like hypertension, tachycardia, arrhythmia, respiratory distress, and tremors develop more frequently, while the most common neuropsychiatric side effects observed are ataxia, hallucinations, confusion, disorientation, meaningless speech and behaviors as well as psychomotor agitation. Sometimes an adverse effect known as acute toxic psychosis occurs very rarely in pediatric patients. ${ }^{3}$ Due to the widespread usage of $1 \%$ cyclopentolate hydrochloride solution as a cycloplegic agent for refractory examinations, it has been reported that several systemic side effects may occur while using solutions more than $0.5 \%$ concentrations in the pediatric patient population ${ }^{4}$ due to the fact that children are extremely prone to systemic toxicity due to their lower body weight as well as the presence of an immature blood-brain barrier for drug metabolism. Moreover, as maximum side effects have been reported while using these drops in infants, therefore it is recommended that infants undergoing retinopathy of prematurity examination should be kept under observation for the next $24 \mathrm{~h}$ after the examination is carried out. ${ }^{5}$

Since the risk of side effects of cyclopentolate hydrochloride is greater at higher concentrations and doses, it can be observed that the side effects of cyclopentolate hydrochloride are not only dose-dependent but can also develop with routine drug usage at an appropriate concentration or dose. ${ }^{6}$ Nevertheless, it was also suggested that the incidence of side effects increased in those patients having a prior systemic disease; ${ }^{7}$ however, this was not applicable in our patient, as he exhibited many side effects without the presence of any systemic disease.

As there are no specific laboratory tests for the diagnosis of the central anticholinergic syndrome; some other drug intoxications such as opioids and benzodiazepines, respiratory disorders, fluid and electrolyte imbalances, hormonal imbalance as well as psychiatric illness could be considered in the differential diagnosis. In our patient, no laboratory tests were conducted due to a high suspicion of drug intoxication. Therefore, it was suggested that if the patient's presenting symptoms did not improve or worsen during the follow-up, then necessary investigations would be carried out. Owing to the fact that other treatment modalities were only indicated when the symptoms of central anticholinergic syndrome either became subjectively evident or a life-threatening situation occurred as in our patient, our patient was provided symptomatic treatment which led to the improvement of the patient's symptoms in about 4 hours. To conclude, although cyclopentolate hydrochloride is a frequently used drug in ophthalmologic examinations, it also has some serious systemic side effects when given in inappropriate doses. Since unrestrained drug administration may cause an increase in the incidence of potential side effects, utmost care should be taken that the drug should always be administered by trained health personnel. As our case report highlighted the importance of correct usage of topical ophthalmic preparations in children, it also proved that although the side effects improved without the need for medical intervention, sometimes an early recognition of systemic toxicity symptoms as well as initiation of required interventions were essential for optimal recovery of patients. It is also recommended that during the application of such drugs, the nasolacrimal duct openings should be closed by pressing the eye's inner corners with a finger to prevent the ophthalmic solution from leaking in the nose, passing through the nasolacrimal duct, and finally to the systemic circulation to avoid any adverse reactions.

\section{Ethics}

Informed Consent: Parents of the patient provided informed consent to publish the report.

Peer-reviewed: Externally peer-reviewed. 


\section{Authorship Contributions}

Surgical and Medical Practices: N.K., F.B., Concept: M.E.U., H.A., Design: N.K., H.A., Data Collection or Processing: Y.G., Analysis or Interpretation: M.E.U., F.B., Literature Search: Y.G., N.K., Writing: N.K., M.E.U., F.B., Y.G., H.A.

Conflict of Interest: The authors declared no potential conflicts of interest with respect to the research, authorship, and/or publication of this article.

Funding: The authors received no financial support for the research, authorship, and/or publication of this article.

\section{REFERENCES}

1. Bhatia SS, Vidyashankar C, Sharma RK, Dubey AK. Systemic toxicity with cyclopentolate eye drops. Indian Pediatr. 2000;37:329-31.
2. V Pooniya, N Pandey. Systemic toxicity of topical cyclopentolate eyedrops in a child. Eye (Lond). 2012;26:1391-2.

3. Derinoz O, Er A. Inability to walk, disequilibrium, incoherent speech, disorientation following the instillation of $1 \%$ cyclopentolate eyedrops: Case report. Pediatr Emerg Care. 2012;28:59-60.

4. Ağın $H$, Gerçek H, Kutlu A, Gülez N, Atlıhan F. Topikal siklopentolat kullanımına bağlı gelişen deliryum tablosu: Bir vaka takdimi. Çocuk Sağlığı ve Hastalıkları Derg. 2008;51:39-42.

5. Laws DE, Morton C, Weindling M, Clark D. Systemic effects of screening for retinopathy of prematurity. Br J Ophthalmol. 1996;80:425-8.

6. Awan KJ. Adverse systemic reactions of topical cyclopentolate hydrochloride. Ann Ophthalmol. 1976;8:695-8.

7. Mirshahi A, Kohnen T. Acute psychotic reaction caused by topical cyclopentolate use for cycloplegic refraction before refractive surgery: case report and review of the literature. J Cataract Refract Surg. 2003;29:102630. 